\title{
Improved Lower Bounds for tree-like Resolution over Linear Inequalities *
}

\author{
Arist Kojevnikov \\ St.Petersburg Department of Steklov Institute of Mathematics \\ 27 Fontanka, 191023 St.Petersburg, Russia \\ http://logic.pdmi.ras.ru/ arist/
}

\begin{abstract}
We continue a study initiated by Krajíček of a Resolutionlike proof system working with clauses of linear inequalities, $\mathrm{R}(\mathrm{CP})$. For all proof systems of this kind Krajíček proved in [1] an exponential lower bound of the form:

$$
\frac{\exp \left(n^{\Omega(1)}\right)}{M^{O\left(W \log ^{2} n\right)}}
$$

where $M$ is the maximal absolute value of coefficients in a given proof and $W$ is the maximal clause width.

In this paper we improve this lower bound. For tree-like R(CP)-like proof systems we remove a dependence on the maximal absolute value of coefficients $M$, hence, we give the answer to an open question from [2]. Proof follows from an upper bound on the real communication complexity of a polyhedra.

Key words: propositional proof complexity, integer programming, cutting planes
\end{abstract}

Many well known methods in an area of pseudo-boolean constraints optimization like a branch-and-bound [3] and Cutting Planes with the deduction rule [4] can be defined in terms of Resolution proof system that operates with clauses of linear inequalities, $\mathrm{R}(\mathrm{CP})$ [1]. This proof system is a natural extension of Resolution and can be viewed as a generalization of Resolution over formulas in $k$-DNF, $\operatorname{Res}(k)$, that was introduced in [5]. In the last few years much attention was paid to complexity of $\operatorname{Res}(k)$ [6-8]. From the other hand, it is not much known about the complexity of $\mathrm{R}(\mathrm{CP})$, while it and similar proof systems are often used in practice [9-11].

Consider a $\mathrm{R}(\mathrm{CP})$-like proof system as a system that work with clauses of linear inequalities using finite set of tautologically valid axioms and sound derivation rules with at most two hypotheses. The main goal of this paper is to improve lower bounds on restricted but still very important family of $\mathrm{R}(\mathrm{CP})$-like proof systems. Namely, we proved better lower bounds for tree-like R(CP)-like proof systems.

\footnotetext{
* Supported in part by Russian Science Support Foundation, INTAS (grant 04-833836) and RFBR (grants 05-01-00932, 06-01-00502). The paper was done during the stay of the author at the Max-Planck-Institut für Mathematik, Bonn, Germany.
} 
The main idea of exponential lower bounds that based on monotone interpolation theorem is a transformation of a proof $P$ of the formula $F$ into a monotone circuit $C$ of size polynomial in $|P|$. If the formula $F$ formalizes that the intersection of two disjoint NP-sets is not empty, then the circuit $C$ separates these two disjoint NP-sets. For example, the pair of disjoint NP-sets, consisting of a set of graphs with a $k$-clique and the set of $(k-1)$-colorable graphs, the monotone circuit that separates one set from another has at least exponential size [12]. Hence, the size of proof $P$ is exponential.

There is a very nice connection between boolean circuits and communication complexity [13], and sometimes it is more easier to think in terms of communication complexity then in terms of circuits. This idea was used by Krajíček to prove many important exponential lower bounds in $[14,1,2]$. He reduced the proofinto-circuit transformation problem into a problem of proving upper bounds on communication complexity of specific decision problems.

In this paper we give an answer to one of the open questions from [2]: we prove new upper bound on real monotone communication complexity of a polyhedra and, hence, a better lower bound for tree-like $\mathrm{R}(\mathrm{CP})$-like proof systems. The proof is straightforward. The basic techniques are the same as in $[14,1,2]$.

The paper is organized as follows. In Sect. 1 we give all necessary definitions, in Sect. 2 we recall the notion of interpolation and prove new lower bound on tree-like $\mathrm{R}(\mathrm{CP})$-like proof systems. In Sect. 3 we discuss related open questions.

\section{Definitions}

In this paper we use the following notation: we typically denote integer vectors with letters $a, b, c$, their coordinates with $a_{i}, b_{i}, c_{i}$, vectors of Boolean variables with $u, v, w, x, y, z$ and integers with $A, B, C$. We will write $a \cdot x$ instead of $\sum_{i} a_{i} x_{i}$

\subsection{Resolution over linear inequalities}

Now we describe several propositional proof systems for the language of systems of linear inequalities that have no 0/1-solutions. A proof system $\mathrm{R}(\mathrm{CP})$ was defined in [1] as follows. The lines of the system are disjunctions of linear inequalities: $a \cdot x \geq A \vee \ldots \vee b \cdot x \geq B$. The derivation rules are (we denote by $\Gamma$ an arbitrary disjunction of linear inequalities)

$$
\begin{array}{ll}
\frac{a \cdot x \geq A \vee \Gamma \quad b \cdot x \geq B \vee \Gamma}{(a+b) \cdot x \geq A+B \vee \Gamma}, & \frac{a \cdot x \geq A \vee \Gamma}{C a \cdot x \geq C A \vee \Gamma}, \quad \text { where } C \geq 0, \\
\frac{C a \cdot x \geq A \vee \Gamma}{a \cdot x \geq\lceil A / C\rceil \vee \Gamma}, \quad \overline{x_{i} \geq 0} & \frac{-x_{i} \geq-1}{\Gamma} \quad \text { for all variables } x_{i}, \\
\overline{a \cdot x \geq A \vee(-a) \cdot x \geq 1-A}, & \frac{\Gamma}{a \cdot x \geq A \vee \Gamma}, \quad \frac{a \cdot x \geq A \vee a \cdot x \geq A \vee \Gamma}{a \cdot x \geq A \vee \Gamma} .
\end{array}
$$

Note that one can omit $0 \geq 1$ from $0 \geq 1 \vee \Gamma$ because the contradiction $0 \geq 1$ is easily transformable into any other inequality. The goal is to derive $0 \geq 1$. 
We also define a family of $\mathrm{R}(\mathrm{CP})$-like proof systems, that operate with disjunctions of linear inequalities by finite set of tautologically valid axioms and sound derivation rules that have at most two hypotheses. We are interested in its sub-family of p-passive $\mathrm{R}(\mathrm{CP})$-like proof systems, where all derivation rules are of the form

$$
\frac{\Delta_{1} \vee \Gamma_{1} \quad \Delta_{2} \vee \Gamma_{2}}{\Delta_{3} \vee \Gamma_{1} \vee \Gamma_{2}}
$$

where $\Delta_{i}$ and $\Gamma_{i}$ are arbitrary disjunctions of linear inequalities and $\left|\Delta_{i}\right| \leq p$, for $i=1,2$.

\subsection{Real Communication Complexity}

The following set of definitions is an extension of boolean communication complexity $[13,15]$, that allows players to communicate with each other not only by bits, but with real numbers. It was introduced in [2].

Let $I$ be finite set, $U, V \subset\{0,1\}^{*}, R \subseteq U \times V \times I$ be such that

$$
\forall u \in U, v \in V \exists i \in I R(u, v, i)
$$

We will call relations satisfying this condition multifunctions.

The following two definition were given in [16].

Definition 1. A real communication protocol $P$ over $U \times V$ with range $I$ is a binary tree where each internal node $v$ is labeled by two function $a_{v}: U \rightarrow \mathbb{R}$, giving player $A$ move, and $b_{v}: V \rightarrow \mathbb{R}$, giving player $B$ move, and each leaf is labeled by an element $i \in I$.

On input $(x, y)$, the players construct a path thought the tree according to the following rule: At each internal node $v$ labeled by $\left(a_{v}, b_{v}\right)$, if $a_{v}(x)>b_{v}(y)$, then the next node is the left son of $v$ and otherwise the right son of $v$. If for every $u \in U$ and $v \in V$ the value $i$ of $P$ satisfies $R(u, v, i)$, we say that $P$ computes $R$.

Definition 2. The real communication complexity of a multifunction $R, C C^{\mathbb{R}}(R)$, is the minimal depth of a real communication protocol $P$, over all $P$ that compute $R$.

Usually, sets $U, V$ are defined by some partial Boolean function $f$ that maps $W \subseteq\{0,1\}^{n}$ to $\{0,1\}$. We take $U:=f^{-1}(1), V:=f^{-1}(0)$ and $I:=\{1, \ldots, n\}$. Relation $R(u, v, i)$ is true if strings $u$ and $v$ differ in position $i$. We are interested in monotone partial Boolean functions, that have at least one extension to a monotone Boolean function [13]. For such a function $f$ define $R_{f}^{\text {mono }} \subseteq U \times V \times I$ by

$$
R_{f}^{\text {mono }}(u, v, i) \quad \text { iff } \quad u \in U \wedge v \in V \wedge u_{i}=1 \wedge v_{i}=0 .
$$

As it happens with monotone boolean functions and Boolean communication complexity, there is a relation between the real communication complexity of $R_{f}^{m o n o}$ and the depth of monotone real circuit computing $f$. 


\subsection{Monotone Real Circuits}

A monotone real circuit is a circuit of fan-in 2 computing with real numbers where every gate computes a nondecreasing real function [17]. Since monotone real circuits are generalization of monotone boolean circuits, we require that they output 0 or 1 on every input from $\{0,1\}^{*}$. The depth and size of the monotone real circuit are defined as for boolean circuits.

Lemma 1 (Lemma 1.4, [2]). Let $f$ be a partial monotone boolean function. Then $C C^{\mathbb{R}}\left(R_{f}^{\text {mono }}\right)$ is at most the minimal depth of a monotone real circuit $C$ that computes the function $f$. Moreover,

$$
C C^{\mathbb{R}}\left(R_{f}^{\text {mono }}\right) \leq \log _{3 / 2} S^{\mathbb{R}}(f),
$$

where $S^{\mathbb{R}}(f)$ is the minimal size of a monotone real formula computing $f$.

There is an important open question about the converse statement. A positive answer on it would immediately imply an extension of lower bound proved in this paper from tree-like $\mathrm{R}(\mathrm{CP})$ to general $\mathrm{R}(\mathrm{CP})[2]$.

\subsection{Local Search Protocols}

The notions of local search protocol and monotone local search protocol were defined in [14] and they generalize the notion of real communication protocol. We need them for transformation of a refutation in some proof system into the real circuit in a natural and intuitive way.

Definition 3 (Definition 2.1, [2]). Let $U, V \subseteq\{0,1\}^{n}$ be two sets and let $R \subseteq U \times V \times I$ be a multifunction. A local search protocol for $R$ is a labeled directed graph $G$ satisfying the following conditions:

1. Graph $G$ is acyclic and has one source denoted by $\emptyset$. The nodes with zero outdegree are leaves, all other are inner nodes. All inner nodes have out-degree 2.

2. All leaves are labeled by elements of $I$.

3. There is a strategy $S(u, v, x)$ that assigns to a node $x$ and a pair $u \in U$ and $v \in V$ one of the two children $S(u, v, x)$ of $y$.

4. For every pair $u \in U, v \in V$ there is a set $F(u, v)$ of nodes of $G$ satisfying:

(a) $\emptyset \in F(u, v)$.

(b) $x \in F(u, v) \rightarrow S(u, v, x) \in F(u, v)$.

(c) If $i$ is the label of a leaf from $F(u, v)$ then $R(u, v, i)$ holds.

We call such set $F$ the consistency condition.

The local search protocol is tree-like iff the underlying graph is a tree.

A local search protocol for a particular multifunction $R=\left\{(u, v, i) \mid u_{i}=\right.$ $\left.1 \wedge v_{i}=0\right\}$ is called a monotone local search protocol for $U, V$. 
Definition 4 (Definition 2.2, [2]). Let $G$ be a local search protocol for $R$. Let $S(u, v, x)$ be the strategy and $F(u, v)$ be the consistency condition of $G$.

The real communication complexity of $G$, denoted $C C^{\mathbb{R}}(G)$, is the minimal $t$ such that for every $x \in G$ the players (first knows pair $(u, x)$, the second knows $(v, x))$ decide $x \in F(u, v)$ and compute $S(u, v, x)$ by real communication protocol by depth at most $t$.

For tree-like local search protocol it is possible to prove an exponential lower bounds on the following set of functions:

Let $I, J$ be sets of size $n$. Consider a monotone Boolean function BPM that gives to a bipartite graph $\Gamma \subseteq I \times J$ the value 1 iff $\Gamma$ contains a perfect matching. Inputs to BPM are $n^{2}$ variables $x_{i j}, i \in I, j \in J$. Their truth evaluations are in one to one correspondence with bipartite graphs.

Theorem 1 (Theorem 2.5, [2]). Let $G$ be a tree-like local search protocol for $B P M$ of size $S$, such that $C C^{\mathbb{R}}(G)=t$. Then

$$
S=\exp \left(\Omega\left(\left(\frac{n}{t \log n}\right)^{1 / 2}\right)\right) .
$$

\section{Lower bound for tree-like R(CP)-like proof systems}

The following definition was introduced in [14] and is a generalization of usual derivation in a proof system. A sequence of sets $D_{1}, \ldots, D_{k} \subseteq\{0,1\}^{N}$ is a semantic derivation of $D_{k}$ from $A_{1}, \ldots, A_{m}$ if each $D_{i}$ is either one of $A_{j}$, or contains $D_{i_{1}} \cap D_{i_{2}}$ for some $i_{1}, i_{2}<i$. Till the end of this section we use $N=$ $n+s+t$. Let us consider the following problem for two players:

Definition 5 (Definition 3.1, [2]). For set $A \subseteq\{0,1\}^{N}$ we fix $u, v \in\{0,1\}^{n}$, $y \in\{0,1\}^{s}$ and $z \in\{0,1\}^{t}$. Consider the following three tasks:

1. Decide whether $(u, y, z) \in A$.

2. Decide whether $(v, y, z) \in A$.

3. If $(u, y, z) \in A$ and $(v, y, z) \notin A$, then find such $i \leq n$ that

$$
u_{i}=1 \wedge v_{i}=0
$$

or find some $u^{\prime}$ satisfying

$$
u^{\prime} \geq u \wedge\left(u^{\prime}, y, z\right) \notin A \quad\left(\text { where } u^{\prime} \geq u \text { means } \bigwedge_{i \leq n}\left(u_{i}^{\prime} \geq u_{i}\right)\right) .
$$

These tasks can be solved by two players, one knowing $(u, y)$ and another one knowing $(v, z)$.

$A$ monotone real communication complexity of $A, M C C^{\mathbb{R}}(A)$ is the minimal $t$ such that tasks 1-3 have real communication complexity at most $t$. 
We define subset $Q(b)$ of $\mathbb{Z}^{W}$ as follows

$$
Q(b)=\left\{a \in \mathbb{Z}^{W} \mid \forall i \leq W\left(a_{i} \leq b_{i}-1\right)\right\} .
$$

We need to prove the following lemma to improve the lower bound for tree-like $\mathrm{R}(\mathrm{CP})$-like proof systems. It extends Lemma 5.1, [14] to real communication complexity.

Lemma 2. Let linear mapping

$$
H:\{0,1\}^{N} \rightarrow \mathbb{Z}^{W}
$$

be defined by a matrix with elements from $\mathbb{Z}$.

Let $Y \subseteq \mathbb{Z}^{W}$ be any set defined as

$$
Y=\mathbb{Z}^{W} \backslash Q(b)
$$

for some $b \in \mathbb{Z}^{W}$. We fix $X:=H^{-1}(Y)$.

Then

$$
M C C^{\mathbb{R}}(X)=O(W)+O(\log (n)) .
$$

Proof. 1. To decide whether $(u, y, z) \in X$ we need to find such $i \in 1, \ldots, W$ that

$$
\sum_{j=1}^{n} h_{i j} \cdot u_{j}+\sum_{j=n+1}^{n+s} h_{i j} \cdot y_{j}+\sum_{j=n+s+1}^{n+s+t} h_{i j} \cdot z_{j} \geq b_{i}
$$

Player $A$ knows all elements in this sum except $z$. Let integer $z_{i}$ satisfy the equality

$$
\sum_{j=1}^{n} h_{i j} \cdot u_{j}+\sum_{j=n+1}^{n+s} h_{i j} \cdot y_{j}+z_{i}=b_{i}
$$

The players compare $z_{i}$ and $z_{i}^{\prime}=\sum_{j=n+s+1}^{n+s+t} h_{i j} \cdot z_{j}$ for all $i \in 1, \ldots, W$ and if for some $i$ the inequality $z_{i} \leq z_{i}^{\prime}$ holds, then (1) is also holds and therefore $(u, y, z) \in X$. Otherwise, $(u, y, z) \notin X$.

To decide whether $(u, y, z) \in X$ players use the real communication protocol of depth $W$.

2. Similarly, by real communication protocol of depth $W$, players can decide whether $(v, y, z) \in X$.

3. Assume that $(u, y, z) \in X$ and $(v, y, z) \notin X$. It means that for some $i \in$ $1, \ldots, W$ is

$$
\sum_{j=1}^{n} h_{i j} \cdot u_{j}+\sum_{j=n+1}^{n+s} h_{i j} \cdot y_{j}+\sum_{j=n+s+1}^{n+s+t} h_{i j} \cdot z_{j} \geq b_{i}
$$

and also

$$
\sum_{j=1}^{n} h_{i j} \cdot v_{j}+\sum_{j=n+1}^{n+s} h_{i j} \cdot y_{j}+\sum_{j=n+s+1}^{n+s+t} h_{i j} \cdot z_{j}<b_{i}
$$


From the last two inequalities it follows that

$$
\sum_{j \in J} h_{i j} \cdot u_{j}>\sum_{j \in J} h_{i j} \cdot v_{j}
$$

where $J=\{1, \ldots, n\}$.

For all $j$ such that $h_{i j}<0$ first player assigns 1 to $u_{j}$. If for some $u^{\prime} \geq u$ the triple $\left(u^{\prime}, y, z\right) \notin X$, then he communicates one bit of the answer to second player, and they stop if it is equal to 1 . Otherwise,

$$
\sum_{j \in J} h_{i j} \cdot u_{j}^{\prime}>\sum_{j \in J} h_{i j} \cdot v_{j}
$$

where $J=\{1, \ldots, n\}$

Let fix $J_{1}=\{1, \ldots,\lfloor n / 2\rfloor\}$ and $J_{2}=\{\lfloor n / 2\rfloor+1, \ldots, n\}$. Note that it is holds either

$$
\sum_{j \in J_{1}} h_{i j} \cdot u_{j}^{\prime}>\sum_{j \in J_{1}} h_{i j} \cdot v_{j} \quad \text { or } \quad \sum_{j \in J_{2}} h_{i j} \cdot u_{j}^{\prime}>\sum_{j \in J_{2}} h_{i j} \cdot v_{j},
$$

otherwise (2) is not satisfying. Continue with one of the satisfied inequalities and find such $j$ that $\left(u_{j}^{\prime}=1 \wedge v_{j}=0\right)$ or $\left(u_{j}^{\prime}=0 \wedge v_{j}=1\right)$. Since in this case, $h_{i j}>0$ (otherwise $u_{j}^{\prime}$ is equal to 1 ), we have that $u_{j}^{\prime}=u_{j}=1 \wedge v_{j}=0$. The real communication complexity of described binary search procedure is equal to $O(\log (n))$.

Following [2] we define a set $\tilde{A}$ for the $A \subseteq\{0,1\}^{n+s}$ as follows:

$$
\tilde{A}:=\bigcup_{(a, b) \in A}\left\{(a, b, c) \mid c \in\{0,1\}^{t}\right\},
$$

where $a, b, c$ are from $\{0,1\}^{n},\{0,1\}^{s}$ and $\{0,1\}^{t}$ respectively. For $B \subseteq\{0,1\}^{n+t}$ we define in the same way $\tilde{B}$ :

$$
\tilde{B}:=\bigcup_{(a, c) \in B}\left\{(a, b, c) \mid b \in\{0,1\}^{s}\right\} .
$$

Theorem 2 (Theorem 3.2, [2]). Let $A_{1}, \ldots, A_{m} \subseteq\{0,1\}^{n+s}$ and $B_{1}, \ldots, B_{\ell} \subseteq$ $\{0,1\}^{n+t}$ be two set families. Assume that there is a semantic derivation $\pi=$ $D_{1}, \ldots, D_{k}$ of the empty set $\emptyset=D_{k}$ from $A_{1}, \ldots, A_{m}, B_{1}, \ldots, B_{\ell}$. Assume also that all the sets $A_{1}, \ldots, A_{m}$ satisfy the following monotone condition:

$$
(u, y) \in \bigcap_{j \leq m} A_{j} \wedge u \leq u^{\prime} \rightarrow\left(u^{\prime}, y\right) \in \bigcap_{j \leq m} A_{j}
$$

and $M C C^{\mathbb{R}}\left(D_{i}\right) \leq t$ for all $i \leq k$. 
Define sets $U$ and $V$ as follows:

$$
U=\left\{u \in\{0,1\}^{n} \mid \exists y \in\{0,1\}^{s} ;(u, y) \in \bigcap_{j \leq m} A_{j}\right\}
$$

and

$$
V=\left\{v \in\{0,1\}^{n} \mid \exists z \in\{0,1\}^{t} ;(v, z) \in \bigcap_{j \leq \ell} B_{j}\right\} .
$$

Then there is a monotone local search protocol $G$ for the sets $U, V$ of size at most $k+n$ with real communication complexity $C C^{\mathbb{R}}$ at most $t$.

Moreover, if the semantic derivation $\pi$ is tree-like, then local search protocol $G$ is also tree-like.

The following theorem extends [2, Theorem 3.3] from CP-like proof systems to $\mathrm{R}(\mathrm{CP})$-like proof systems.

Theorem 3. Let a system of linear inequalities $E_{1}(x, y), \ldots, E_{m}(x, y), F_{1}(x, z)$, $\ldots, F_{\ell}(x, z)$ contain only variables $\left(x_{1}, \ldots, x_{n}\right),\left(y_{1}, \ldots, y_{s}\right)$ and $\left(z_{1}, \ldots, z_{t}\right)$. Assume that there is a refutation $\pi$ of the system in $\mathrm{R}(\mathrm{CP})$-like proof system with $k$ lines. Let every clause in $\pi$ have at most $W$ occurrences of linear inequalities. Assume also that $x_{i}$ occur in all $E_{1}, \ldots, E_{m}$ only with non-negative coefficients.

Then there is a monotone local search protocol $G$ for $U, V$ :

$$
\begin{aligned}
& U=\left\{u \in\{0,1\}^{n} \mid \exists y \in\{0,1\}^{s} ;(u, y) \text { satisfying } \bigwedge_{i \leq m} E_{i}(u, y)\right\}, \\
& V=\left\{v \in\{0,1\}^{n} \mid \exists z \in\{0,1\}^{t} ;(v, z) \text { satisfying } \bigwedge_{j \leq \ell} F_{j}(v, z)\right\}
\end{aligned}
$$

such that the size of $G$ is at most $k+n$ and its real communication complexity is $O(W)+O(\log (n))$.

Moreover, if the refutation $\pi$ is tree-like, then local search protocol $G$ is also tree-like.

Proof. Consider a clause $D=\left\{h_{i} \cdot(x, y, z)^{T} \geq b_{i} \mid i \leq W\right\}$ in the refutation $\pi$. Then assignment $(x, y, z)$ satisfies it iff

$$
H \cdot(x, y, z) \in \mathbb{Z}^{W} \backslash Q\left(\left(b_{1}, \ldots, b_{W}\right)\right),
$$

where $H$ is a $N \times W$-matrix with strings $h_{i}$. Replace each clause $D$ in $\pi$ by $\tilde{D} \subseteq\{0,1\}^{N}$ of assignments satisfying it to obtain a semantic refutation of $\tilde{E}_{i}$ and $\tilde{F}_{i}$. By Lemma 2 for every set $S$ occurring in the refutation it holds that $M C C^{\mathbb{R}}(S)=O(W)+O(\log (n))$. To complete the proof apply Theorem 2 .

\subsection{Exponential Lower Bounds}

In [2] the following set of inequalities was introduced, $\mathrm{Hall}_{n}$, that formalize Hall's theorem.

Let $|I|=|J|=n$. 
1. $\sum_{i} y_{k i} \geq 1$, for all $1 \leq k \leq n$.

2. $y_{k i}+y_{k^{\prime} i} \leq 1$, for all $1 \leq k<k^{\prime} \leq n$.

3. $\sum_{j} y_{k j}^{\prime} \geq 1$, for all $1 \leq k \leq n$.

4. $y_{k j}^{\prime}+y_{k^{\prime} j}^{\prime} \leq 1$, for all $1 \leq k<k^{\prime} \leq n$.

5. $y_{k j}^{\prime}+y_{k i}-x_{i j} \leq 1$, for all $1 \leq k \leq n, i \in I, j \in J$.

Let $E_{i}\left(x, y, y^{\prime}\right)$ be all these linear inequalities. Note, that the set

$$
U:=\left\{x \in\{0,1\}^{n^{2}} \mid \exists y, y^{\prime}\left(\bigwedge_{i} E_{i}\left(x, y, y^{\prime}\right)\right)\right\}
$$

determines a set of graphs with BPM equal to 1 .

The set $V$ of graphs with BPM equal to 0 can be defined analogously by inequality system $F_{j}\left(x, z, z^{\prime}\right)$. The union set of all inequalities $E_{i}$ and $F_{j}$ is denoted by $\mathrm{Hall}_{n}$.

Theorem 4. Let $\pi$ be a tree-like refutation of $\mathrm{Hall}_{n}$ in any $\mathrm{R}(\mathrm{CP})$-like proof system. Then $|\pi| \geq \exp \left(\Omega\left(\left(\frac{n}{W \log (n)+(\log (n))^{2}}\right)^{1 / 2}\right)\right)$.

Proof. By Theorem 3 there is a tree-like monotone local search protocol $G$ for BPM problem of size $k+n$ and real communication complexity $t=O(W)+$ $O(\log (n))$. The required lower bound follows from Theorem 1 .

\section{Open questions}

In this section we formulate some important open questions.

1. Remove a dependence on maximal absolute value of coefficient in Krajíček's exponential lower bound for general $\mathrm{R}(\mathrm{CP})$-like proof system or prove that it is impossible.

2. Remove a dependence on maximal number of inequalities in clauses for treelike $\mathrm{R}(\mathrm{CP})$-like proof systems or prove that it is impossible.

Remark 1 (Iddo Tzameret). We cannot remove a dependence on maximal number of inequalities in clauses for general $\mathrm{R}(\mathrm{CP})$-like proof systems, since in [18] it was proved that $\operatorname{Res}(2)$ (a subsystem of $\mathrm{R}(\mathrm{CP})$ with polynomially bounded coefficients) does not have monotone interpolation in almost exponential time.

\section{Acknowledgments}

The author is very grateful to Dima Grigoriev, Jan Krajíček, Alexander S. Kulikov and Iddo Tzameret for helpful comments and is indebted to Edward A. Hirsch for enlightening discussions. 


\section{References}

1. Krajíček, J.: Discretely ordered modules as a first-order extension of the cutting planes proof system. Journal of Symbolic Logic 63(4) (1998) 1582-1596

2. Krajíček, J.: Interpolation by a game. Mathematical Logic Quarterly 44(40) (1998) 450-458

3. Land, H., Doig, A.G.: An automatic method for solving discrete programming problems. Econometrica 28 (1960) 497-520

4. Bonet, M., Pitassi, T., Raz, R.: Lower bounds for cutting planes proofs with small coefficients. The Journal of Symbolic Logic 62(3) (1997) 708-728

5. Krajíček, J.: On the weak pigeonhole principle. Fundamenta Mathematicæ 170(13) (2001) 123-140

6. Atserias, A., Bonet, M.L., Esteban, J.L.: Lower bounds for the weak pigeonhole principle and random formulas beyond resolution. Information and Computation 176(2) (2002) 136-152

7. Segerlind, N., Buss, S.R., Impagliazzo, R.: A Switching Lemma for Small Restrictions and Lower Bounds for k-DNF Resolution. SIAM Journal on Computing 33(5) (2004) 1171-1200

8. Alekhnovich, M.: Lower bounds for k-DNF resolution on random 3-CNFs. In: STOC '05: Proceedings of the thirty-seventh annual ACM symposium on Theory of computing, New York, NY, USA, ACM Press (2005) 251-256

9. Prestwich, S.: Incomplete dynamic backtracking for linear pseudo-boolean problems. Annals of Operations Research 130 (2004) 57-73

10. Chai, D., Kuehlmann, A.: A fast pseudo-boolean constraint solver. IEEE Trans. on CAD of Integrated Circuits and Systems 24(3) (2005) 305-317

11. Manquinho, V.M., Marques-Silva, J.: On using cutting planes in pseudo-boolean optimization. Journal of Satisfiability, Boolean Modeling and Computation 2 (2006) 209-219

12. Razborov, A.A.: Lower bounds on the monotone complexity of some Boolean functions. Dokl. Akad. Nauk SSSR 281(4) (1985) 798-801 In Russian: English translation in Soviet Math. Dokl. 31:354-357, 1985.

13. Karchmer, M., Wigderson, A.: Monotone circuits for connectivity require superlogarithmic depth. SIAM Journal on Discrete Mathematics 3(2) (1990) 255-265

14. Krajíček, J.: Interpolation theorems, lower bounds for proof systems, and independence results for bounded arithmetic. Journal of Symbolic Logic 62(2) (1997) $457-486$

15. Kushilevitz, E., Nisan, N.: Communication Complexity. Cambridge University Press (1997)

16. Bonet, M.L., Esteban, J.L., Galesi, N., Johannsen, J.: On the relative complexity of resolution refinements and cutting planes proof systems. SIAM J. Comp. 30(5) (2000) 1462-1484

17. Pudlák, P.: Lower bounds for resolution and cutting plane proofs and monotone computations. Journal of Symbolic Logic 62(3) (1997) 981-998

18. Atserias, A., Bonet, M.L.: On the automatizability of resolution and related propositional proof systems. Information and Computation 189(2) (2004) 182-201 